\title{
A germline alteration of $E R B B 2$ increases the risk of breast cancer in Chinese Han women with a familial history of malignant tumors
}

\author{
YAN JU $^{1 *}$, LIFENG WANG ${ }^{2 *}$, SHENGJUN TA $^{3}$, RUI SHU ${ }^{1}$, SHANLING YANG ${ }^{1}$, \\ XICAN GAO ${ }^{1}$, HONGPING SONG ${ }^{1}$ and LIWEN LIU ${ }^{1}$ \\ ${ }^{1}$ Department of Ultrasonic Medicine, Xijing Hospital; ${ }^{2}$ Department of Biochemistry and Molecular Biology, \\ The Fourth Military Medical University, Xi'an, Shaanxi 710032; ${ }^{3}$ Department of Ultrasound, \\ Yan'an People's Hospital, Yan'an, Shaanxi 716000, P.R. China
}

Received June 8, 2018; Accepted June 7, 2019

DOI: $10.3892 / 01.2019 .10646$

\begin{abstract}
Previous studies have demonstrated that a family history of breast cancer is considered a risk factor, and hereditary factors may be involved in breast cancer pathogenesis. Next-generation sequencing techniques were used to analyze 111 cancer-associated genes in patients with breast cancer with a familial history of malignant tumors in the pre-experiment and a novel variant, receptor tyrosine-protein kinase erbB-2 (ERBB2) c.338G $>$ A: p.R113Q was identified in two cases of breast cancer. $E R B B 2$ is considered an important oncogene, and overexpression or mutation of the ERBB2 gene may lead to the occurrence or metastasis of tumors. To assess a potential association between rs185670819 and breast cancer, 117 patients with breast cancer and a familial history of any cancer, who were diagnosed by experienced pathologists at the Xijing Hospital (Shaanxi, China) between July 2015 and December 2016, were recruited. The presence of the missense variant was confirmed using bi-directional Sanger sequencing of samples from the patients with breast cancer and 250 healthy controls. The effects of the missense mutation on the structure and function of $E R B B 2$ were analyzed in silico. The missense variant, R113Q, in patients with breast cancer with a familial history of malignant tumors in China, was present in 8 patients [6.8\% (95\% CI: 3.21-13.45)] and 3 of 250 healthy controls [1.2\% (95\% CI: 0.31-3.76; OR=6.04, 95\% CI: 1.573-23.214, $\mathrm{P}=0.009)]$. Of the 8 patients with the $\mathrm{R} 113 \mathrm{Q}$ variant, 6 patients had a family history of cancer of the digestive system. The
\end{abstract}

Correspondence to: Dr Hongping Song or Dr Liwen Lu, Department of Ultrasonic Medicine, Xijing Hospital, The Fourth Military Medical University, 172 Chang Le Road, Xi'an, Shaanxi 710032, P.R. China

E-mail: song.hp@foxmail.com

E-mail: liuliwen@fmmu.edu.cn

*Contributed equally

Key words: breast cancer, potential risk factor, receptor tyrosine-protein kinase erbB-2-R113Q present study suggests that $E R B B 2$ c.338G $>$ A: p.R113Q may be a potential risk factor in the development and progression of breast cancer.

\section{Introduction}

Breast cancer is the most common malignancy and the leading cause of cancer-associated mortality in women worldwide (1). Genetic alterations serve a critical role in the etiology of cancer, disrupting gene function and increasing the risk of cancer (2). Germline mutations in the BRCA1 and BRCA2 genes are the most common causes of breast cancer susceptibility, with $B R C A 1$ conferring a risk of breast cancer of $\sim 67 \%$ and $66 \%$ for $B R C A 2$, by the age of 80 (3). Although other moderate-to-low-penetrance genes, such as CHEK2, PALB2 and $A T M$, serve an important role in breast cancer predisposition (4), BRCA1/2 mutations account for $10 \%$ of all cases in the Chinese population (5).

Previous studies have indicated that a family history of other types of cancer, such as colon cancer, prostate cancer, and ovarian cancer, is associated with an increased risk of breast cancer occurrence (6-8). However, studies on the presence of germline mutations in patients with breast cancer with a family history of tumors are limited, to the best of our knowledge. To determine a comprehensive spectrum of genetic factors for patients with breast cancer with a family history of malignant tumors in the Chinese population, targeted exome sequencing was performed (the judgment standard of next-generation-sequencing is shown in Appendix S1) and 111 cancer-associated genes from 13 patients with breast cancer with a family history of malignant tumors were identified. In addition, a novel allele, ERBB2 c.338G >A: p.R113Q that was enriched in two cases of breast cancer was identified.

Receptor tyrosine-protein kinase ebb $(H E R / E R B B) 2$ is part of the $E R B B$-like oncogene family and is considered a critical oncogene, mutations of which may initiate the onset of breast cancer. HER2 is capable of forming a homodimer on its own or heterodimers with other receptors, including HER1 and HER3, which results in the autophosphorylation of tyrosine residues of the kinase domains. These reactions subsequently trigger intracellular signaling cascades, including mitogen-activated protein kinase, phosphoinositide 3-kinase, protein kinase $\mathrm{C}$ 
and signal transducer and activator of transcription, which primarily promote cell proliferation and prevent apoptosis (9).

Next-generation sequencing studies have demonstrated that somatic mutations in $E R B B 2$ are present in a considerable number of tumors, including in breast cancer $(10,11)$. Overexpression gene editing experiments have designated a number of these mutations as activating and oncogenic elements, that is, acting as carcinogens $(11,12)$. Therefore it is hypothesized that $E R B B 2$ c.338G $>$ A: p.R113Q may be a genetic risk factor of breast cancer. The aim of the present study was to sequence the loci and determine whether there was an association between rs185670819 and breast cancer risk.

\section{Materials and methods}

Patients. A total of 117 patients with breast cancer with a familial history of cancer, all of whom were diagnosed by experienced pathologists at The Xijing Hospital (Shaanxi, China) were recruited for the present study between July 2015 and December 2016. The inclusion criteria were as follows: i) Women aged between 18 and 74 years old with primary breast cancer, regardless of histological type or stage; and ii) patients had at least one first-degree, or two second-degree relatives with cancer (no limitation on tumor type). Exclusion criteria were as follows: i) Patients with breast cancer with no familial history of cancer; and ii) patients with breast cancer without informed consent forms. Within the same time period, a total of 250 healthy volunteers were included in the study as a control group. The mean ages of the case and control groups were $52.5 \pm 9.7$ and $52.1 \pm 9.9$ years, respectively. All participants enrolled in our research were from unrelated families and provided written informed consent. The Ethics Committee of Xijing Hospital approved the study protocol. Peripheral blood samples were drawn and stored in EDTA-coated tubes at $-80^{\circ} \mathrm{C}$.

DNA extraction and genotyping. DNA was extracted from peripheral blood using commercially available kits, in accordance with the manufacturer's protocol (E.Z.N.A. ${ }^{\circledR}$ Blood DNA Mini kit, Omega Bio-Tek, Norcross, GA, USA). A 300 bp fragment within the extracellular domain of $E R B B 2$ was amplified using PCR. Primer sequences were: Forward 5'-ACGTGCTCA TCGCTCACAAC-3' and reverse 5'-CCCAGAAGGGACACC ATTTC-3'. The PCR product was confirmed using electrophoresis in a $1.5 \%$ agarose gel and subsequently purified using the E.Z.N.A. ${ }^{\circledR}$ Gel Extraction kit (Omega Bio-Tek). The purified PCR product sequencing was performed with a BigDye Terminator v3.1 cycle sequencing kit (Applied Biosystems; Thermo Fisher Scientific, Inc., Waltham, MA, USA) on the 3730xl DNA Analyzer system (Applied Biosystems; Thermo Fisher Scientific, Inc.), and the data obtained were analyzed with the 3730xl Genetic Analyzer Data Collection Software v3.0 (Applied Biosystems; Thermo Fisher Scientific, Inc.).

In silico analysis of ERBB2 encoded by the ERBB2 c.338G $>$ A variant. The scores of SIFT (13), PolyPhen v2 (14), MutationTaster (15), and GEPR++ (16) was collected from dbNSFP (version 2.6) (17) to predict the possible impact of an amino acid substitution on the structure and the function of HER2. PyMOL 2.0 software (https://pymol.org/2/) (18) was used for the analysis of the 3D structure of HER 2 encoded by
Table I. Clinical and pathological characteristics of patients with breast cancer.

\begin{tabular}{|c|c|}
\hline Characteristic & Value \\
\hline Mean age $\pm \mathrm{SD}$, years & $52.5 \pm 9.7$ \\
\hline \multicolumn{2}{|l|}{ Stage, n (\%) } \\
\hline I & $34(29.1)$ \\
\hline IIA & $44(37.6)$ \\
\hline IIB & $14(12.0)$ \\
\hline IIIA & $21(17.9)$ \\
\hline IIIC & $3(2.6)$ \\
\hline IV & $1(0.9)$ \\
\hline \multicolumn{2}{|l|}{ Tumor size, $\mathrm{n}(\%)$} \\
\hline$\leq 2 \mathrm{~cm}$ & $47(40.2)$ \\
\hline$>2 \mathrm{~cm}$ & $70(59.8)$ \\
\hline \multicolumn{2}{|l|}{ Lymph nodes, n (\%) } \\
\hline Negative & $67(57.2)$ \\
\hline Positive & $50(42.8)$ \\
\hline \multicolumn{2}{|l|}{ Distant metastasis, n (\%) } \\
\hline Negative & $116(99.1)$ \\
\hline Positive & $1(0.9)$ \\
\hline \multicolumn{2}{|l|}{ Pathology, n (\%) } \\
\hline Invasive ductal carcinoma & $103(88)$ \\
\hline Ductal carcinoma in situ & $8(6.8)$ \\
\hline Invasive lobular carcinoma & $2(1.7)$ \\
\hline Invasive mucinous carcinoma & $2(1.7)$ \\
\hline Invasive medullary carcinoma & $2(1.7)$ \\
\hline \multicolumn{2}{|l|}{ HER2 expression, $\mathrm{n}(\%)$} \\
\hline Negative (score 0 and 1 ) & $54(46.2)$ \\
\hline Uncertain (score 2) & $25(21.4)$ \\
\hline Positive (score 3 ) & $27(23.1)$ \\
\hline Unknown & $11(9.4)$ \\
\hline
\end{tabular}

HER2, receptor tyrosine-protein kinase erbB-2.

rs185670819. Conservation analysis among a range of vertebrate species was performed using data obtained from University of California Santa Cruz Genome Browser [Human Dec. 2013 (GRCh38/hg38) assembly; http://genome.ucsc.edu] (19).

Statistical analysis. A $\chi^{2}$ test was used to assess the differences in genotype frequencies between cases and controls. A one-sided P-value and odds ratios (OR) with $95 \%$ confidence interval $(95 \% \mathrm{CI})$ were calculated to demonstrate an association between the allelic ERBB2 and breast cancer risk. $\mathrm{P}<0.05$ was considered to indicate a statistically significant difference. The Hardy-Weinberg equilibrium (HWE) test was performed to confirm the samples are representative of the population according to a method previously described (20).

\section{Results}

Study population. To examine whether ERBB2 c.338G>A: p.R113Q was a potential risk factor for patients with breast 
Table II. Variant of ERBB2 present in Chinese patients with breast cancer.

\begin{tabular}{lccccccc}
\hline Base change & $\begin{array}{c}\text { Protein } \\
\text { change }\end{array}$ & $\begin{array}{c}\text { Variant } \\
\text { class }\end{array}$ & Genotype & $\begin{array}{c}\text { Frequency in Frequency in } \\
\text { patients (\%) }\end{array}$ & $\begin{array}{c}\text { OR } \\
\text { controls (\%) }\end{array}$ & $\begin{array}{c}\text { P-value } \\
(95 \% \text { CI) }\end{array}$ & $\begin{array}{c}\text { Pardy-weinberg } \\
\text { equilibrium }\end{array}$ \\
\hline $\begin{array}{l}\text { NM_001005862.2: } \\
\text { c.338G }>\text { A }\end{array}$ & $\begin{array}{c}\text { p.Arg } \\
\text { 113Gln }\end{array}$ & Missense & heterozygous & $8 / 117(6.8)$ & $3 / 250(1.2)$ & 0.009 & 6.04 \\
\end{tabular}

ERBB2, receptor tyrosine-protein kinase erbB-2; OR, odds ratio; CI, confidence interval.

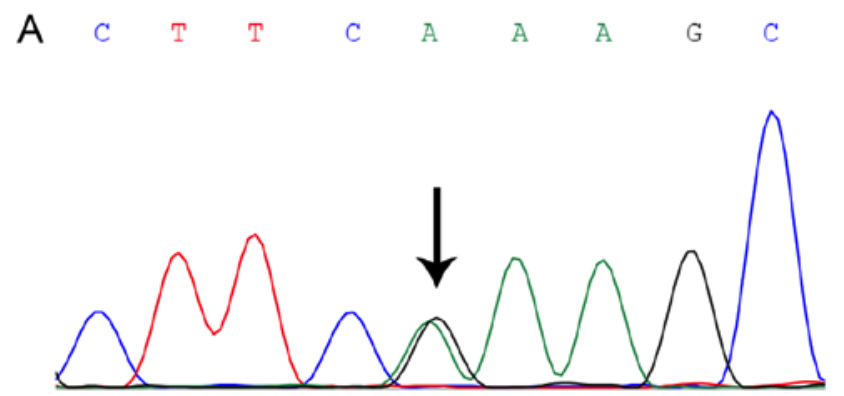

B

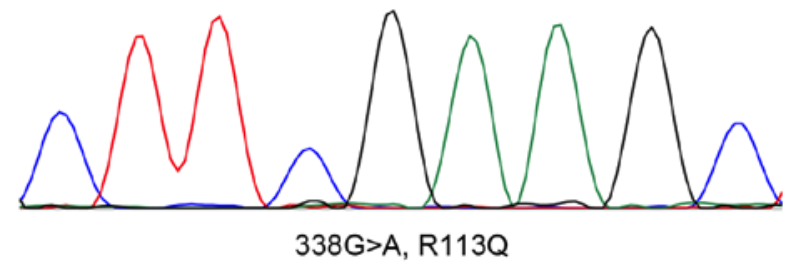

Figure 1. R113Q allele identified in the ERBB2 gene. (A) The wild-type sequences of $E R B B 2$. (B) R113Q allele detected in the present sudy: $\mathrm{G} \rightarrow \mathrm{A}$ substitution $(338 \mathrm{G}>\mathrm{A}$, black arrow). ERBB2, receptor tyrosine-protein kinase erbB-2.

cancer with a familial history of malignant tumors, ERBB2 c. $338 \mathrm{G}>\mathrm{A}$ was sequenced using the DNA isolated from peripheral blood samples of 117 Chinese women with breast cancer. Both the case and control groups consisted of individuals from the Han ethnic community of China, and there were no relationships between the recruited patients. The clinical and pathological characteristics of the patients with breast cancer are presented in Table I. The majority of the subjects had early-stage breast cancer (stage I, stage IIA and stage IIB); accounting for $78.6 \%$ of the overall participants, and $99.1 \%$ of the patients had no distant metastasis. The histological type of invasive ductal carcinoma accounted for $88 \%$, which was the most common among the recruited patients. Almost one-half of the patients with breast cancer were negative for HER2 expression $(n=54,46.2 \%)$.

ERBB2 c.338G $>$ A: p.R113Q variant. The $\mathrm{R} 113 \mathrm{Q}$ variant that was detected in the present study (Table II; Fig. 1) resulted in an amino acid alteration from arginine to glutamine in the receptor L domain of ERBB2. All the R113Q carriers had heterozygous genotypes (Table II). The frequency of R113Q occurrence was significantly higher in patients with breast cancer with a family history of tumors $(8 / 117,6.8 \%)$ compared with the healthy group $\left(3 / 250,1.2 \% ; \chi^{2}=8.712, P=0.003\right.$; Table II). The risk of breast cancer increased significantly with the occurrence of R113Q carriers $(\mathrm{OR}=6.04 ; 95 \% \mathrm{CI}$ 1.573-23.214, $\mathrm{P}=0.009$; Table II). There was no deviation in the HWE of R113Q $(\mathrm{P}=0.70)$, which indicated the population was representative.

Additionally, the results of the 1,000 Genomes Project indicated that the frequency of occurrence of this allele accounts for $1.9 \%$ of the Han people from Beijing and the south of China (Table III), a figure similar to our finding (1.2\%). Furthermore, the R113Q allele was present at a lower frequency in Japanese populations and was not detected at all in Europeans (Table III) (21).

Clinical characteristics of the carriers. Out of the eight carriers with family histories of cancer, six of the patients had at least one relative with tumors of the digestive system. The rate of R113Q-associated breast cancer with a family history of tumors of the digestive system was $75 \%$ (Table IV). In the present study, the R113Q was more prevalent in patients with breast cancer who did not present with amplification of ERBB2 (Table IV).

In silico analysis of the R113Q mutation. The R113Q variant in the $E R B B 2$ gene was predicted to be involved in the extracellular ligand-binding domain, a domain that is highly conserved in biological evolution. The mutation-induced structural change of the extracellular domain was thus hypothesized to cause a deficit in function. The arginine at residue 113 of ERBB2 was highly conserved among different vertebrates (Fig. 2) where a red box indicates residue 113 of HER 2 in each of the vertebrates. Although the predicted effects of the R113Q mutation from four different programs were inconsistent, two of the in silico tools predicted its pathogenicity, although specific details were not provided (Table V). The results of the 3D structure suggested that the alteration of the amino acid at position 113 (Fig. 3) led to a structural change in the side chain of ERBB2. Accordingly, the R113Q variant was predicted to have a potentially damaging effect on the function of the protein, the mechanism of which will be investigated in future studies.

\section{Discussion}

In the present study a novel susceptible locus allele, ERBB2 c.338G $>$ A: p.R113Q, which may increase breast cancer risk 


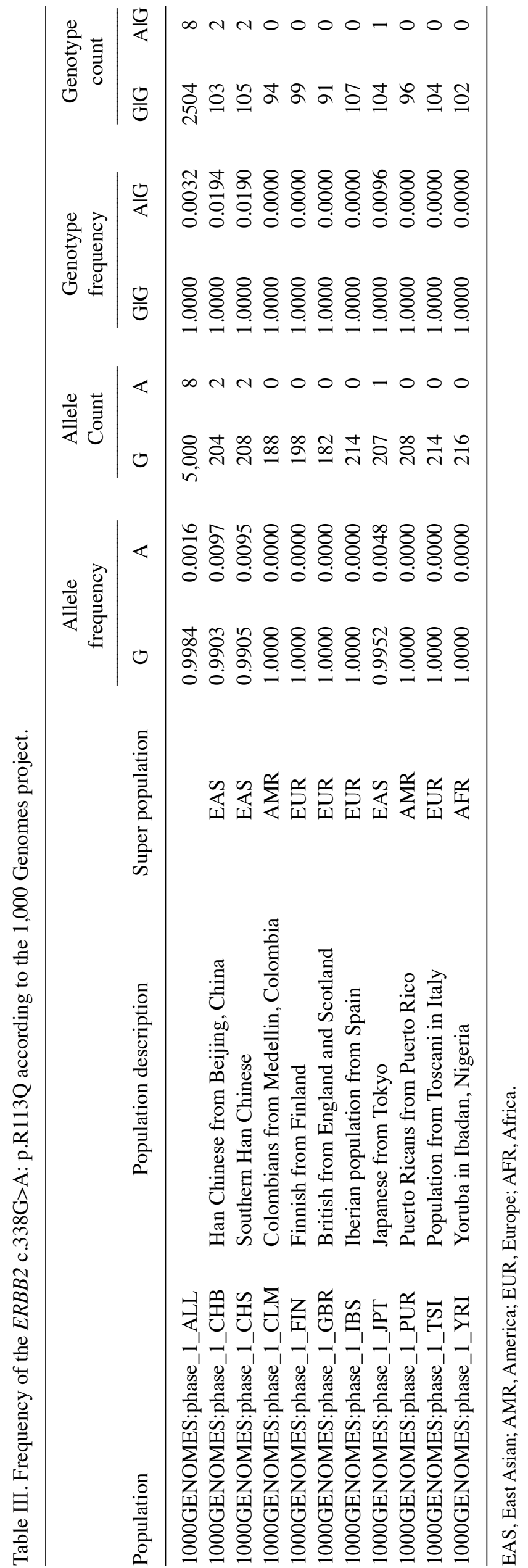

in Chinese women with a family history of the tumor, was identified. The R113Q was detected in $6.8 \%$ of the patients with familial history of malignant tumors, increasing the risk of breast cancer 6.04-fold. The association between the R113Q variant and the risk of breast cancer in Chinese women with a familial history of malignant tumors was reported for the first time, to the best of our knowledge. In silico analysis of the R113Q variant suggested that the R113Q variant may exhibit a potentially pathogenic effect. The single nucleotide polymorphism was additionally more prevalent in patients with breast cancer with a familial history of different types of cancer of the digestive system.

The data presented in the present study support previous studies which have demonstrated an ERBB2 polymorphism as a potential risk factor for the development of breast cancer, including the best investigated mutation, I655V (rs1136201), which conferred a 1.1-2.3-fold risk of developing breast cancer (22-24). Yamamoto et al (25) additionally demonstrated the potential oncogenicity of a mutation in HER2 (V659E), which caused hereditary lung adenocarcinomas in a Japanese family. The present data, therefore, provide a new marker to determine an increased risk of breast cancer in individuals.

The 113th residue of HER2 is located on the receptor L domain of the extracellular domains (ECDs). Dimerization of the extracellular domains (ECDs) results in phosphorylation of the intracellular domains and initiates signal transduction of the ERBBs. A previous study demonstrated that HER2 can only be activated without a ligand through structural alterations when HER2 is overexpressed (26), with the ECD playing an important role in the dimerization process $(27,28)$. Another study additionally demonstrated that a mutation in the ECDs results in the loss of dimerization and subsequent phosphorylation (29).

R113Q is located on the L domain of the ECDs and is a highly conserved amino acid across the listed vertebrates. As the relationship between the mutation and disease severity had not been established, software was used to predict the effects of the amino acid substitution on protein function, and also generate the three-dimensional shape of the protein coded by ERBB2. Mutation Taster, GEPR++, and assessment of the three-dimensional structure all predicted the mutation at this locus to be potentially pathogenic.

A limitation of the present study is that the identification of the R113Q allele was from a small cohort. It is necessary to expand the cohort in the future to include larger samples of the breast cancer patient population, as well as to identify any potential link(s) that may have remained undetected with the relatively small sample size used. In vivo models, such as transgenic mice, to further investigate the molecular mechanisms of the $E R B B 2$ variation at extracellular domains may be required.

The present study identified a novel R113Q allele that may contribute to an increased risk of breast cancer in the Chinese population with a family history of tumors, especially tumors of the digestive system. The presence of the R113Q allele was highlighted in $6.8 \%$ of the patient cohort, conferring a 6.04-fold increased risk of breast cancer compared to non-carriers. The structure of the mutant $E R B B 2$ was predicted in silico to have a potential pathogenic effect in breast cancer. Therefore R113Q may be a novel susceptibility factor for Chinese women with a family history of tumors. 
Table IV. Characteristics of the 8 patients with breast cancer who carried the ERBB2 c.338G>A: p.R113Q variant.

\begin{tabular}{lccccl}
\hline ID & Age at diagnosis & ER & PR & HER2 & \multicolumn{1}{c}{ Family cancer history } \\
\hline 20150828001 & 37 & - & - & - & $\begin{array}{l}\text { Paternal uncle, EC; paternal uncle, EC; } \\
\text { paternal cousin, EC; father, GC }\end{array}$ \\
20151014001 & 38 & - & - & + & Mother, SC; father, SC \\
20151223001 & 42 & + & + & + & Father, EC \\
201602170134 & 76 & + & + & - & Maternal uncle, SC; maternal uncle, LC \\
201603080153 & 56 & - & - & - & Father, SC; grandson, LK \\
201606080274 & 60 & + & + & - & Mother, BC \\
201610090471 & 50 & - & - & + & Father, LV; sister, LK \\
F0017BRCA201609020028 & 51 & + & + & - & Father, LC
\end{tabular}

ERBB2, receptor tyrosine-protein kinase erbB-2; BC, breast cancer; EC, esophageal cancer; SC, stomach cancer; LC, lung cancer; LK, leukemia; LV, liver cancer; GC, gastric cancer.

Table V. In silico prediction outcomes of R113Q.

\begin{tabular}{|c|c|c|c|c|c|}
\hline Variation & Variation ID & SIFT (score) & PolyPhen_2 (score) & Mutation Taster (score) & GERP++ (score) \\
\hline p.Arg 113Gln & rs185670819 & Tolerated $(0.061)$ & Benign (0.162) & Disease causing (1) & Conserved (5.21) \\
\hline
\end{tabular}

$\begin{array}{ll}\text { Human } & \text { RELQLR SLTE } \\ \text { Rhesus } & \text { RELQLR SLTE } \\ \text { Mouse } & \text { RELQLR SLTE } \\ \text { Dog } & \text { RELQLR SLTE } \\ \text { Elephant } & \text { RELQLR SLTE } \\ \text { Zebrafish } & \text { RELQLR SLTE }\end{array}$

Figure 2. Conservation of the amino acid sequence of ERBB2 in the extracellular domain among six vertebrae species.

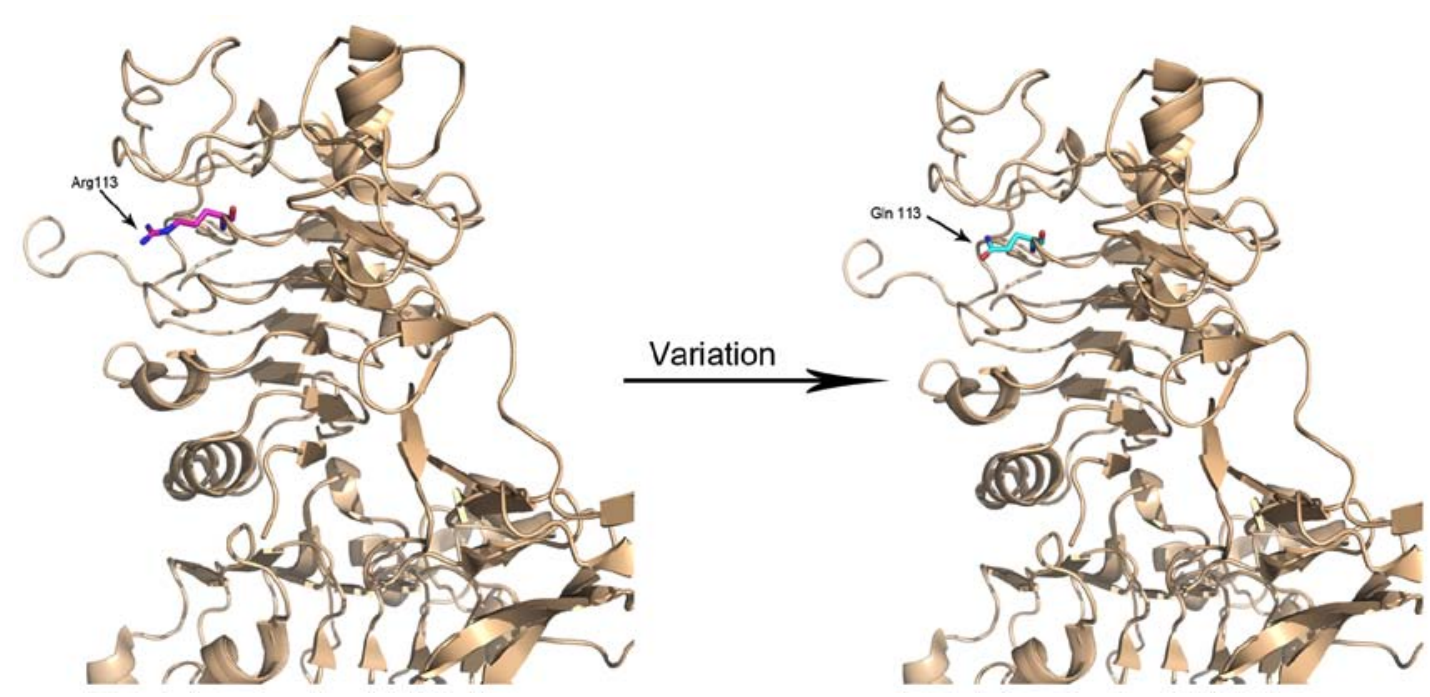

Figure 3. Predicted 3D structure of an amino-acid alteration from Arg to Gln at residue 113 of ERBB2. Arg, arginine; Gln, glutamine. 


\section{Acknowledgements}

Not applicable.

\section{Funding}

The present study received funding from The National Natural Science Foundation of China (grant no. 81401416), The Shaanxi International Scientific and Technological Cooperation and Exchange Program (Shaanxi, China; grant no. 2017KW-057), 2017 Shaanxi Scientific Research Projects in Higher Education (grant no. XGH17281) and 2016 Key Project of Clinical High and New Technology of Xijing Hospital (Shaanxi, China).

\section{Availability of data and materials}

The datasets used and/or analyzed during the present study are available from the corresponding author on reasonable request.

\section{Authors' contributions}

HS and LL participated in the design of the study and wrote the original protocol for the research. YJ and LW conducted the experiments, analyzed the data, and drafted the manuscript. ST analyzed the data and revised the manuscript. RS, SY, and XG collected the data and revised the manuscript. All authors directly provided their contribution, read and approved the final manuscript.

\section{Ethics approval and consent to participate}

The Ethics Committee of Xijing Hospital (Shaanxi, China) approved the present study. All participants enrolled in the present study provided written informed consent.

\section{Patient consent for publication}

Not applicable.

\section{Competing interests}

The authors declare that they have no competing interests.

\section{References}

1. Ferlay J, Soerjomataram I, Dikshit R, Eser S, Mathers C, Rebelo M, Parkin DM, Forman D and Bray F: Cancer incidence and mortality worldwide: Sources, methods and major patterns in GLOBOCAN 2012. Int J Cancer 136: E359-E386, 2015.

2. Kleibl Z and Kristensen VN: Women at high risk of breast cancer: Molecular characteristics, clinical presentation and management. Breast 28: 136-144, 2016.

3. Kuchenbaecker KB, Hopper JL, Barnes DR, Phillips KA, Mooij TM, Roos-Blom MJ, Jervis S, van Leeuwen FE, Milne RL, Andrieu N, et al: Risks of breast, ovarian, and contralateral breast cancer for BRCA1 and BRCA2 mutation carriers. JAMA 317 2402-2416, 2017.

4. Economopoulou P, Dimitriadis G and Psyrri A: Beyond BRCA: New hereditary breast cancer susceptibility genes. Cancer Treat Rev 41: 1-8, 2015

5. Liede A and Narod SA: Hereditary breast and ovarian cancer in Asia: Genetic epidemiology of BRCA1 and BRCA2. Hum Mutat 20: 413-424, 2002.

6. Negri E, Braga C, La Vecchia C, Franceschi S and Parazzini F: Family history of cancer and risk of breast cancer. Int J Cancer 72 735-738, 1997.
7. Parazzini F, La Vecchia C, Negri E, Franceschi S and Tozzi L: Family history of breast, ovarian and endometrial cancer and risk of breast cancer. Int J Epidemiol 22: 614-618, 1993.

8. Zhou W, Ding Q, Pan H, Wu N, Liang M, Huang Y, Chen L, Zha X, Liu X and Wang S: Risk of breast cancer and family history of other cancers in first-degree relatives in Chinese women: A case control study. BMC Cancer 14: 662, 2014.

9. Schettini F, Buono G, Cardalesi C, Desideri I, De Placido S and Del Mastro L: Hormone receptor/human epidermal growth factor receptor 2-positive breast cancer: Where we are now and where we are going. Cancer Treat Rev 46: 20-26, 2016.

10. Ellis MJ, Ding L, Shen D, Luo J, Suman VJ, Wallis JW, Van Tine BA, Hoog J, Goiffon RJ, Goldstein TC, et al: Whole-genome analysis informs breast cancer response to aromatase inhibition. Nature 486: 353-360, 2012.

11. Bose R, Kavuri SM, Searleman AC, Shen W, Shen D, Koboldt DC, Monsey J, Goel N, Aronson AB, Li S, et al: Activating HER2 mutations in HER2 gene amplification negative breast cancer. Cancer Discov 3: 224-237, 2013.

12. Greulich H, Kaplan B, Mertins P, Chen TH, Tanaka KE, Yun CH, Zhang X, Lee SH, Cho J, Ambrogio L, et al: Functional analysis of receptor tyrosine kinase mutations in lung cancer identifies oncogenic extracellular domain mutations of ERBB2. Proc Natl Acad Sci USA 109: 14476-14481, 2012.

13. Kumar P, Henikoff S and Ng PC: Predicting the effects of coding non-synonymous variants on protein function using the SIFT algorithm. Nat Protoc 4: 1073-1081, 2009.

14. Adzhubei I, Jordan DM and Sunyaev SR: Predicting functional effect of human missense mutations using PolyPhen-2. Curr Protoc Hum Genet 7: Unit7 20, 2013.

15. Schwarz JM, Cooper DN, Schuelke M and Seelow D: MutationTaster2: Mutation prediction for the deep-sequencing age. Nat Methods 11: 361-362, 2014.

16. DavydovEV,GoodeDL,Sirota M,Cooper GM,Sidow A and Batzoglou S: Identifying a high fraction of the human genome to be under selective constraint using GERP++. PLoS Comput Biol 6: e1001025, 2010.

17. Liu X, Jian X and Boerwinkle E: dbNSFP v2.0: A database of human non-synonymous SNVs and their functional predictions and annotations. Hum Mutat 34: E2393-E2402, 2013.

18. DeLano WL: PyMOL: An open-source molecular graphics tool. CCP4 Newsletter On Protein Crystallography 40: 82-92, 2002.

19. Kent WJ, Sugnet CW, Furey TS, Roskin KM, Pringle TH, Zahler AM and Haussler D: The human genome browser at UCSC. Genome Res 12: 996-1006, 2002.

20. Wigginton JE, Cutler DJ and Abecasis GR: A note on exact tests of Hardy-Weinberg equilibrium. Am J Hum Genet 76: 887-893, 2005.

21. 1000 Genomes Project Consortium, Auton A, Brooks LD, Durbin RM, Garrison EP, Kang HM, Korbel JO, Marchini JL, McCarthy S and McVean GA, Abecasis GR: A global reference for human genetic variation. Nature 526: 68-74, 2015.

22. Tao W, Wang C, Han R and Jiang H: HER 2 codon 655 polymorphism and breast cancer risk: A meta-analysis. Breast Cancer Res Treat 114: 371-376, 2009.

23. Lu S, Wang Z, Liu H and Hao X: HER2 Ile655Val polymorphism contributes to breast cancer risk: Evidence from 27 case-control studies. Breast Cancer Res Treat 124: 771-778, 2010.

24. Millikan R, Eaton A, Worley K, Biscocho L, Hodgson E, Huang WY, Geradts J, Iacocca M, Cowan D, Conway K and Dressler L: HER2 codon 655 polymorphism and risk of breast cancer in African Americans and whites. Breast Cancer Res Treat 79: 355-364, 2003.

25. Yamamoto H, Higasa K, Sakaguchi M, Shien K, Soh J, Ichimura K, Furukawa M, Hashida S, Tsukuda K, Takigawa N, et al: Novel germline mutation in the transmembrane domain of HER2 in familial lung adenocarcinomas. J Natl Cancer Inst 106: djt338, 2014.

26. Brennan PJ, Kumagai T, Berezov A, Murali R and Greene MI: HER2/Neu: Mechanisms of dimerization/oligomerization. Oncogene 21: 328, 2002.

27. Penuel E, Akita RW and Sliwkowski MX: Identification of a region within the ErbB2/HER2 intracellular domain that is necessary for ligand-independent association. J Biol Chem 277: 28468-28473, 2002.

28. Chantry A: The kinase domain and membrane localization determine intracellular interactions between epidermal growth factor receptors. J Biol Chem 270: 3068-3073, 1995.

29. Hu S, Sun Y, Meng Y, Wang X, Yang W, Fu W, Guo H, Qian W, Hou S, Li B, et al: Molecular architecture of the ErbB2 extracellular domain homodimer. Oncotarget 6: 1695-1706, 2015.

This work is licensed under a Creative Commons Attribution-NonCommercial-NoDerivatives 4.0 International (CC BY-NC-ND 4.0) License. 